\title{
The tendency to recreate ancestral CG dinucleotides in the human genome
}

Mingkun $\mathrm{Li}^{1} \mathrm{2}^{*}$, Su-Shing Chen ${ }^{1}$

\begin{abstract}
Background: The CG dinucleotides are known to be deficient in the human genome, due to a high mutation rate from 5-methylated CG to TG and its complementary pair CA. Meanwhile, many cellular functions rely on these CG dinucleotides, such as gene expression controlled by cytosine methylation status. Thus, CG dinucleotides that provide essential functional substrates should be retained in genomes. How these two conflicting processes regarding the fate of CG dinucleotides - i.e., high mutation rate destroying CG dinucleotides, vs. functional processes that require their preservation remains an unsolved question.

Results: By analyzing the mutation and frequency spectrum of newly derived alleles in the human genome, a tendency towards generating more CGs was observed, which was mainly contributed by an excess number of mutations from CA/TG to CG. Simultaneously, we found a fixation preference for CGs derived from TG/CA rather than CGs generated by other dinucleotides. These tendencies were observed both in intergenic and genic regions. An analysis of Integrated Extended Haplotype Homozygosity provided no evidence of selection for newly derived CGs.

Conclusions: Ancestral CG dinucleotides that were subsequently lost by mutation tend to be recreated in the human genome, as indicated by a biased mutation and fixation pattern favoring new CGs that derived from TG/CA.
\end{abstract}

\section{Background}

DNA methylation is central to important biological processes, including $\mathrm{X}$ chromosome inactivation[1], genetic imprinting [2], gene-expression regulation [3-5], and the defense mechanisms against parasitic DNA and transposons [6,7]. DNA methylation also plays an important role in pathological processes such as cancer [8], is closely related with histone modification and RNAassociated silencing [9], and may be involved in genomic instability [10].

DNA methylation in mammals is carried out by three methyltransferases (DNMT1, DNMT3A, DNMT3B)[11] that target the cytosine in CG dinucleotides. A stochastic model for the maintenance of methylation during DNA replication has been proposed that allows accurate epigenetic inheritance[9,11]. However, CG dinucleotides

\footnotetext{
* Correspondence: mingkun_li@eva.mpg.de

'CAS-MPG Partner Institute of Computational Biology, Shanghai Institutes of Biological Sciences, Chinese Academy of Sciences, 200000, Shanghai, PR China

Full list of author information is available at the end of the article
}

also experience a high mutation rate from cytosine to thymine through the deamination of 5-methylcytosine and a less-than-perfect repair mechanism for resulting G/T mismatches [12-14], which calls into question the stability of methylation of CG dinucleotides as an important epigenetic marker.

In the human genome, the number of CG dinucleotides is $\sim 25 \%$ of that expected given observed base frequencies, which is due to a high mutation rate from 5-methylated CG to TG and its complementary pair CA [15]. $15 \%$ of all CGs are clustered in so called "CpG islands", which are often located at the 5 ' end of genes and overlap with the promoter region, especially in housekeeping genes [16]. Their methylation status is highly associated with gene expression [3], but despite this apparent functional constraint, loss of CpG islands has been observed as a common event in mammalian genomes $[15,17,18]$.

Moreover, multiple pathways exist that preserve CG dinucleotides in the genome, including a lack of methylation of CGs in CpG islands and a strong correction 
bias towards C/G following T/G mismatches $[14,19,20]$. Two enzymes (TDG and MBD4) have been found to selectively remove the thymine from a T:G mismatch in the context of CpG dinucleotides [21,22].

In our previous study [23], we observed a strong fixation bias in favor of derived C/G alleles at CG-related sites (dinucleotides that differ by one-step mutation from CG: CT, CA, CC, AG, TG, GG) compared with C/ $\mathrm{G}$ alleles at CG-free sites (positions not preceded by $\mathrm{C}$ or followed by G). TG and CA, which are predominant at CG-related sites, were suspected to contribute this bias. Since a large fraction of TG/CA sites are originally derived from deamination of of methylated CGs [24], we hypothesize that recreating ancestral CGs may be the driving force behind this bias. Data released by the HapMap project and various genome projects afford us the opportunity to test this hypothesis.

By analyzing the mutation and frequency spectrum of different derived alleles, while taking the sequence context into account, a significant mutation and fixation preference towards transition-generated CGs (CG derived from CA or TG) was observed, while no such preference was observed for transversion-generated CGs (CG derived from CC, GG, CT, AG). This mutation and fixation preference enables the recreation of ancestral CG dinucleotides lost via hypermutation to CA/TG.

\section{Methods}

\section{Data collection}

Human SNP data were retrieved from dbSNP (ftp://ftp. ncbi.nih.gov/snp/, Build 124), and curated by the method described elsewhere[25]. Briefly, the following SNPs were selected: nonindel, biallelic, uniquely mapped to nonrepetitive sequences, validated, and with at least 100 nucleotides (nt) of flanking sequence available both upstream and downstream from the SNP.

Sequences and reads for multiple species were retrieved from the NCBI Nucleotide Databasehttp:// www.ncbi.nih.gov/sites/entrez?db=nuccore and Traces Databasehttp://www.ncbi.nih.gov/Traces. Finally, the chimpanzee(Pan troglodytes, whole genome, 2.82G), gorilla(Gorilla, 9.54G, unassembled), orangutan (Pongo, 18.37G, unassembled), and Gibbon(Nomascus, 18.64G, unassembled) genome sequences were used as outgroups to infer the ancestral allele for the human SNP.

The allele frequencies at each SNP site were estimated from flat files downloaded from the International HapMap Project (http://hapmap.ncbi.nlm.nih.gov/downloads/frequencies/, Build 23). The derived-allele frequency (DAF) for each SNP was estimated in three populations (unrelated individuals only): Yoruba(YRI), Utah residents with ancestry from northern and western Europe(CEU), Japanese and Chinese(ASN); only those
SNPs successfully genotyped in all chromosomes (120, 120,180 respectively) were kept for further analysis.

\section{Inferring ancestral states of the SNPs}

Inferring ancestral states using parsimony may be unreliable if the mutation or substitution rate is high[26]. In mammalian genomes, CG dinucleotides have a very high mutation rate [27]; therefore, two methods were applied to ensure accurate inference of the ancestral state.

First, MegaBLAST was used to map the human SNPs and their flanking sequences(100 bp on each side) to the four outgroup sequences (chimpanzee, gorilla, orangutan, gibbon)[28]. We inferred the ancestral allele only when the chimpanzee, gorilla, orangutan and gibbon alleles were identical and matched one of the human SNP alleles. A PERL script was written to retrieve the human SNPs and their corresponding outgroup alleles from the MegaBLAST result.

Second, we also downloaded a dataset of $14.3 \mathrm{Mb}$ of well curated human/chimpanzee/baboon DNA alignments (http://pbil.univ-lyon1.fr/datasets/MeunierDuret2004/data)[29] and the corresponding human/ rhesus sequences from http://genome.ucsc.edu. Blast was used to construct new alignments, and the final alignment length was shortened to $9.4 \mathrm{Mb}$. Human SNPs that map to the aligned chimp/baboon/rhesus sequences were retrieved and the ancestral state inferred as described above. Given the branch lengths estimated by Steiper and Young [30] for the primate phylogeny, reliable inference of the ancestral state can be obtained using these three outgroups even at CG related positions (Additional file 1).

For $\mathrm{CpG}$ islands, a single outgroup (chimpanzee) was used to infer the mutation direction to maximize the sample size, and the frequency spectrum was then corrected by Hernadez et al.'s method (Additional file 2) [26].

\section{Identification of SNPs located in CpG islands}

We identified 37,503 CpG islands in the human genome using the CGi130 program developed by Takai and Jones (http://cpgislands.usc.edu/)[31,32]. The following search criteria, considered stringent according to the authors, were used: i) GC content $\geq 55 \%$, ii) ObsCG/ ExpCG $\geq 0.65$, and iii) length $\geq 500 \mathrm{bp}$.

\section{Gene annotation}

The gene annotation for each SNP in our study was retrieved from the ENSEMBL database (ftp://ftp. ensembl.org/pub/, version 45), and SNPs were classified as intergenic, intronic, coding, 5'UTR, 3'UTR, 5 'upstream, or 3'downstream. Any SNP classified into more than one category was removed from the analysis. 
This left us with 915243 intergenic, 634809 intronic, 18333 coding, 2055 5'UTR, 11329 3'UTR, 38398 5'upstream and 40749 3'downstream SNPs.

\section{Detection of positive selection}

A Long-Rang Haplotype test was utilized to detect recent positive selection. Specifically, the Integrated Haplotype Score (iHS) was calculated for each SNP, as described elsewhere[33]. We used the suggested cut-off value, namely an extreme positive (iHS $>2$ ) or negative (iHS $<-2)$ iHS score (indicating a longer haplotype associated with the ancestral or the derived allele, respectively), as an indication of recent positive selection.

\section{Recombination hotspots and coldspots}

The locations of recombination hotspots and coldspots were obtained from a fine-scale genetic map estimated from patterns of genetic variation, which provides a kilobase-scale resolution of recombination rates [34,35].

\section{Results}

\section{Mutation and fixation bias towards recreating ancestral} CGs

Following our previous observation of a strong fixation bias in favor of derived $\mathrm{C} / \mathrm{G}$ alleles at CG-related sites [23], we re-examined the mutation and fixation pattern of CG dinucleotides on a genome-wide scale, while taking the sequence context into consideration. The CGs generated by a single mutation from other dinucleotides were classified into transition-generated CGs (Tsg, CG derived from $\mathrm{CA} / \mathrm{TG}$ ) and transversion-generated CGs (Tvg, CG derived from CC/CT/AG/GG). Correspondingly, the dinucleotides derived by mutations from CG are divided into transition-damaged CGs (Tsd, CA/TG derived from CG) and transversion-damaged CGs (Tvd, $\mathrm{CC} / \mathrm{CT} / \mathrm{AG} / \mathrm{GG}$ derived from CG). For comparison, the same mutations located in other sequence contexts (not preceded by $\mathrm{C}$ or followed by $\mathrm{G}$ ) were used as the control data for each of these mutation types. Analyses were also done separately for $\mathrm{CpG}$ islands and non-CpG islands, since they have very different mutation patterns and nucleotide composition.

In the non- $\mathrm{CpG}$ island region, the number of mutations in each annotation category varies substantially, ranging from 819 for transversion-damaged CGs to 19631 for transition-generated CGs for YRI population (Table 1; Additional file 3 for CEU and Additional file 4 for ASN). A total of 9293 mutations are from CG to other dinucleotides, among which $91 \%$ are to CA/TG, which is significantly larger than the fraction of transitions for the control data $(62 \%, \mathrm{P}<2.2 \mathrm{e}-16$, Fisher Exact test). There are 26391 mutations generating new CGs from other dinucleotides, of which $74 \%$ are transitions (derived from $\mathrm{CA} / \mathrm{TG}$ ), which is significantly larger
Table 1 Number of SNPs and average derived-allele frequency (DAF) for different mutation types in different annotation categories

\begin{tabular}{cccccccccc}
\hline & \multicolumn{3}{c}{ Non-CpG island region } & \multicolumn{3}{c}{ CpG island region } \\
\cline { 2 - 9 } $\begin{array}{c}\text { Mutation } \\
\text { type* }\end{array}$ & Intergenic & \multicolumn{2}{c}{ Genic } & \multicolumn{3}{c}{ Intergenic } & \multicolumn{2}{c}{ Genic } \\
\cline { 2 - 9 } & SNPs & DAF & SNPs & DAF & SNPs & DAF & SNPs & DAF \\
\hline All & 88447 & 0.324 & 79241 & 0.317 & 1053 & 0.34 & 3120 & 0.316 \\
Tsd & 3752 & 0.314 & 4722 & 0.303 & 158 & 0.301 & 461 & 0.3 \\
C-Tsd & 22539 & 0.311 & 19525 & 0.303 & 286 & 0.324 & 861 & 0.309 \\
Tsg & 10235 & 0.353 & 9396 & 0.344 & 100 & 0.406 & 240 & 0.373 \\
C-Tsg & 21567 & 0.33 & 19866 & 0.326 & 138 & 0.401 & 403 & 0.339 \\
Tvd & 419 & 0.333 & 400 & 0.322 & 66 & 0.312 & 196 & 0.325 \\
C-Tvd & 14100 & 0.317 & 11667 & 0.309 & 140 & 0.337 & 461 & 0.3 \\
Tvg & 3415 & 0.306 & 3345 & 0.304 & 50 & 0.385 & 169 & 0.309 \\
C-Tvg & 12481 & 0.33 & 10935 & 0.319 & 108 & 0.346 & 354 & 0.302 \\
\hline
\end{tabular}

* The symbols used here are identical to that in the text, with the prefix of Cdenoting the corresponding control dataset.

than the fraction of transitions at control positions (64\%, $\mathrm{P}<2.2 \mathrm{e}-16$, Fisher Exact test). Such a biased process, dominated by mutations between CGs and CAs/ TGs, predicts an increase in CG dinucleotides in the non-CpG island region, and differs significantly from the trend demonstrated by the control data (67831:64859, $\mathrm{P}<2.2 \mathrm{e}-16$, Fisher Exact test).

In the $\mathrm{CpG}$ island region, a different pattern was found: 559 CGs are generated by mutations from other dinucleotides while 881 CGs are destroyed by mutations; there is thus a trend toward decreasing the number of CGs. The trend is in accordance with that shown by the control data for CpG island regions $(1003 \mathrm{Cs} / \mathrm{Gs}$ generated vs. $1748 \mathrm{Cs} / \mathrm{Gs}$ destroyed, $\mathrm{P}=0.139$, Fisher Exact test), but differs significantly from that in the non-CpG island region (26391 CGs generated vs. 9293 CGs destroyed, $\mathrm{P}<2.2 \mathrm{e}-16$, Fisher Exact test).

Derived-allele frequencies (DAF) are shown in Figure 1 for YRI (Additional file 5 for CEU, Additional file 6 for $\mathrm{ASN})$. In the non-CpG island regions, when the derived allele was not preceded by $\mathrm{C}$ or followed by $\mathrm{G}$, transition-generated CGs (Tsg) and transversion-generated CGs (Tvg) had a similar average DAF in the control data (0.328 vs. $0.325, \mathrm{P}=0.1019$, Mann-Whitney $\mathrm{U}$ test), indicating no intrinsic DAF difference for these two types of mutations (A/T- > G/C and C/G/T/A$>\mathrm{G} / \mathrm{C} / \mathrm{G} / \mathrm{C})$. However, the average $\mathrm{DAF}$ of transitiongenerated CGs (Tsg) was significantly greater than for the control data $(0.349$ vs. $0.328, \mathrm{P}=7.6 \mathrm{e}-12$, MannWhitney U test), whereas transversion-generated CGs (Tvg) had a significantly lower average DAF than for the control data ( 0.325 vs. $0.306, \mathrm{P}=1.3 \mathrm{e}-8$, Mann-Whitney $\mathrm{U}$ test). Overall, it suggests a higher probability of fixation for transition-generated CGs (Tsg) than for transversion-generated CGs $(\mathrm{Tvg})(\mathrm{P}<2.2 \mathrm{e}-16$, 


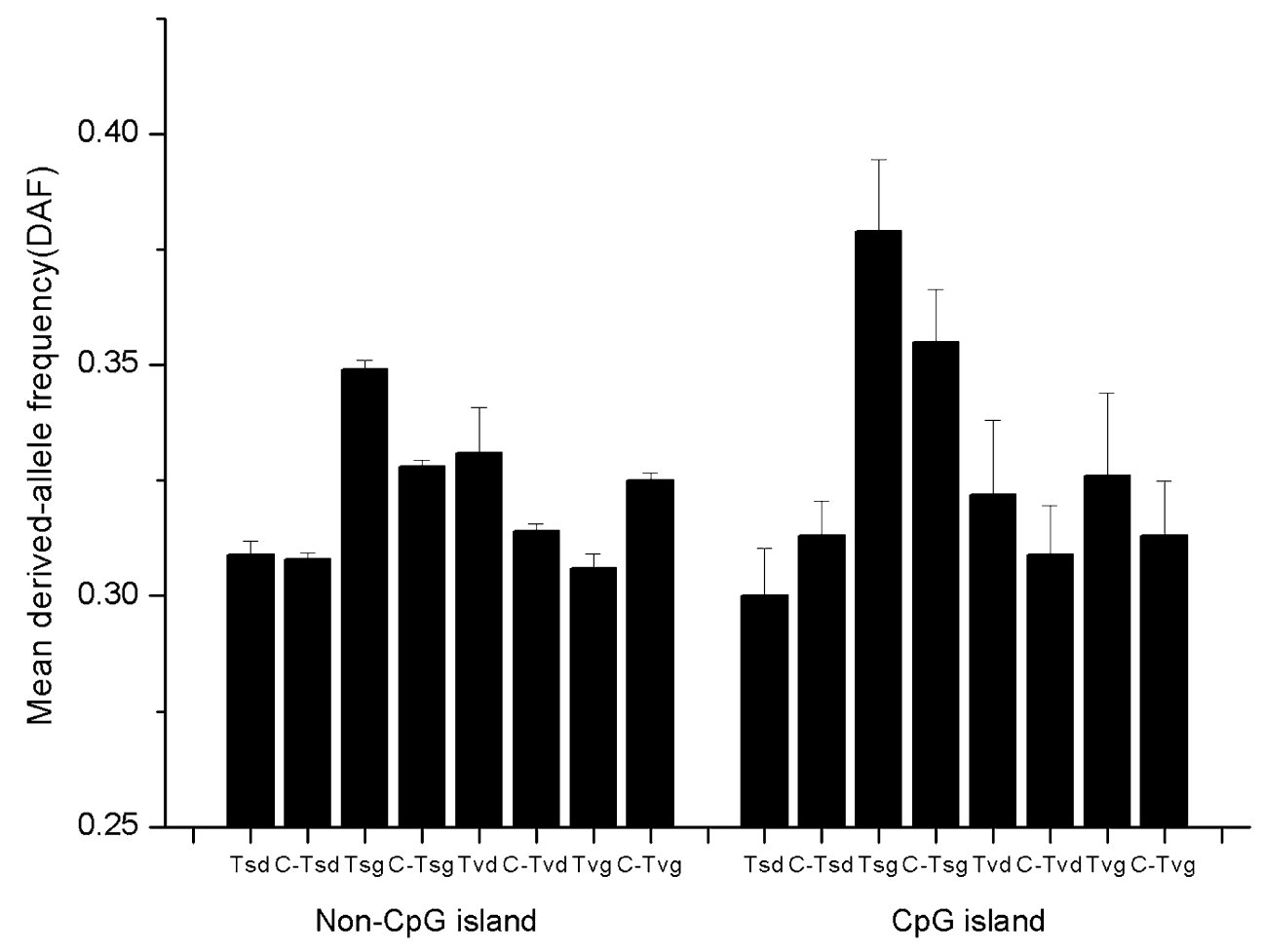

Figure 1 Derived-allele frequency for different mutation types in YRI. Left histogram is for non-CpG island regions, right histogram is for $\mathrm{CPG}$ island regions. Abbreviations are the same as in the text, with the prefix of C-denoting the corresponding control dataset.

Mann-Whitney U test). Meanwhile, the average DAF of CGs destroyed by transitions (i.e., CAs/TGs derived from CGs) did not differ significantly from the control data (0.309 vs. $0.308, \mathrm{P}=0.65$, Mann-Whitney $\mathrm{U}$ test), nor was the average DAF of CGs destroyed by transversions (i.e., CC/GG/CT/AG derived from CGs) significantly different from the control data $(0.331$ vs 0.314 , P $=0.65$, Mann-Whitney U test).

For CpG islands, the DAF for YRI is shown in Figure 1, and in Additional file 5 for CEU and Additional file 6 for ASN. Transition-generated CGs (Tsg) had a much higher DAF than transversion-generated CGs (Tvg)( 0.379 vs. 0.326 for YRI, $P=0.023$, Mann-Whitney U test). However, the control data showed the same trend ( 0.355 vs. $0.313, \mathrm{P}=0.0058$, Mann-Whitney U test).

To investigate the influence of potential errors in assigning ancestral alleles on the above analyses, 9.4 Mb multiple sequence alignments of human/chimpanzee/ baboon/rhesus were created by another method and analyzed. The same mutation and fixation patterns were observed (Additional file 7, Additional file 8).

\section{Selection on the CG dinucleotides?}

To investigate if the above results might reflect selection on functional elements, the SNPs were classfied into four categories: intergenic non-CpG island; genic non$\mathrm{CpG}$ island; intergenic $\mathrm{CpG}$ island; and genic CpG island (genic regions included the sites annotated as intronic, coding, 5'UTR, 3'UTR, 5'upstream, 3'downstream). Normally, intergenic, non-CpG island regions are regarded as neutral, so if selection is playing a role in the trends we observed, there should be differences between genic and intergenic patterns. However, we found similar patterns in genic and intergenic regions (Table 1).

We also applied the iHs test for signals of recent positive selection on newly-generated CG dinucleotides. Newly-generated CGs with iHs $<-2$ may be under positive selection while whose iHs $>2$ may be under negative selection. Among 16843 transition-generated CGs, there were $322(1.9 \%)$ with iHs $<-2$, which is not significantly different from the fraction of transitiongenerated $\mathrm{Cs} / \mathrm{Gs}$ in the control data with $\mathrm{iHs}<-2(2.1 \%$, 
Table 2 Recent selection on the newly generated CG dinucleotides in different mutation categories

\begin{tabular}{|c|c|c|c|c|c|c|c|c|c|c|c|c|}
\hline \multirow[b]{2}{*}{ Population } & \multicolumn{3}{|c|}{ Transition-generated CG } & \multicolumn{3}{|c|}{ Control for transition-generated CG } & \multicolumn{3}{|c|}{ Transversion-generated CG } & \multicolumn{3}{|c|}{ Control for transversion-generated CG } \\
\hline & $\begin{array}{l}\text { Proportion } \\
\text { indicating positive } \\
\text { selection }^{1 .}\end{array}$ & $\begin{array}{l}\text { DAF of the } \\
\text { positive } \\
\text { selected } \mathrm{CG}^{2} \text {. }\end{array}$ & $\begin{array}{l}\text { general } \\
\text { DAF }^{3 .}\end{array}$ & $\begin{array}{l}\text { Proportion } \\
\text { indicating } \\
\text { positive selection }\end{array}$ & $\begin{array}{l}\text { DAF of } \\
\text { the } \\
\text { selected } \\
\text { CG }\end{array}$ & $\begin{array}{l}\text { general } \\
\text { DAF }\end{array}$ & $\begin{array}{l}\text { Proportion } \\
\text { indicating } \\
\text { negative } \\
\text { selection }\end{array}$ & $\begin{array}{l}\text { DAF of the } \\
\text { negative } \\
\text { selected } \mathrm{CG}^{2} \text {. }\end{array}$ & $\begin{array}{l}\text { general } \\
\text { DAF }\end{array}$ & $\begin{array}{l}\text { Proportion } \\
\text { indicating } \\
\text { negative selection }\end{array}$ & $\begin{array}{l}\text { DAF of } \\
\text { the } \\
\text { selected } \\
\text { CG }\end{array}$ & $\begin{array}{l}\text { general } \\
\text { DAF }\end{array}$ \\
\hline YRI & 0.019 & 0.354 & 0.370 & 0.021 & 0.348 & 0.353 & 0.021 & 0.319 & 0.333 & 0.021 & 0.338 & 0.349 \\
\hline CEU & 0.015 & 0.425 & 0.412 & 0.016 & 0.446 & 0.403 & 0.022 & 0.375 & 0.387 & 0.026 & 0.353 & 0.399 \\
\hline ASN & 0.014 & 0.465 & 0.428 & 0.016 & 0.452 & 0.418 & 0.024 & 0.345 & 0.407 & 0.026 & 0.368 & 0.414 \\
\hline
\end{tabular}

1. Proportion of the SNPs whose $\mathrm{iHs}<-2$ (positive selection) or $\mathrm{iHs}>2$ (negative selection) among all SNPs;

2. DAF for SNPs with $\mathrm{iHs}<-2$ (positive selection) or $\mathrm{iHs}>2$ (negative selection);

3. DAF for all SNPS. 
$\mathrm{P}=0.1462$, Fisher's Exact test). Similar results were obtained for transversion-generated CGs (Table 2). Overall, there is no evidence that newly-generated CGs are under recent positive selection.

With the phylogeny of $(((((\mathrm{CG}, \mathrm{CA}) \mathrm{CA}) \mathrm{CA}) \mathrm{CA}) \mathrm{CG})$ CG) for (((((human1,human2)chimpanzee)gorilla)orangutan)gibbon)rhesus)(phylogeny tree is shown in Additional file 9), phylogenetic analyses can reveal situations where an ancestral CG mutated to CA and then back to CG in the human lineage. A total of 34 SNPs were identified as back-mutations to CGs, of which three had a significant iHs value (Table 3 ), which is higher than the fraction of mutations with significant iHs values in the control data ( $P=0.011$, Chi-square test). All three significant iHs values involve transition-generated CGs rather than transversion-generated CGs.

\section{Fixation bias in the recombination hotspots and coldspots}

BGC (Biased Gene Conversion) is a phenomenon universal in vertebrate genomes and results in fixation bias
Table 3 Recent selection on the backmutated CG dinucleotides

\begin{tabular}{cccccccccc}
\hline & & \multicolumn{4}{c}{ YRI } & \multicolumn{2}{c}{ CEU } & \multicolumn{2}{c}{ ASN } \\
\cline { 3 - 9 } SNP ID & $\begin{array}{c}\text { Mutation } \\
\text { type }\end{array}$ & Position & iHs & DAF & iHs & DAF & iHs & DAF \\
\hline rs9409314 & ATG-> & intergenic & 2.1 & 0.8 & 2.39 & 0.339 & 2.56 & 0.545 \\
& ACG & & & & & & & \\
rs7977620 & CAT- $>$ & NAV3 & 1.14 & 0.921 & 2.37 & 0.558 & 2.14 & 0.6 \\
rs4687991 & CGT & upstream & & & & & & & \\
& $\begin{array}{c}\text { CAT- }> \\
\text { CGT }\end{array}$ & intergenic & -0.97 & 0.788 & 2.15 & 0.458 & 1.47 & 0.649 \\
& CGT & & & & & & & & \\
\hline
\end{tabular}

towards the $\mathrm{C} / \mathrm{G}$ allele in the process of repair of double-strand breaks induced by recombination[36]. If the BGC effect is responsible for the fixation preference observed here (namely, transition-generated CGs are more prone to be fixed in the genome compared with the control data), then recombination hotspots and coldspots should differ. The result is shown in Figure 2 for YRI (Additional file 10 for CEU, Additional file 11 for ASN).

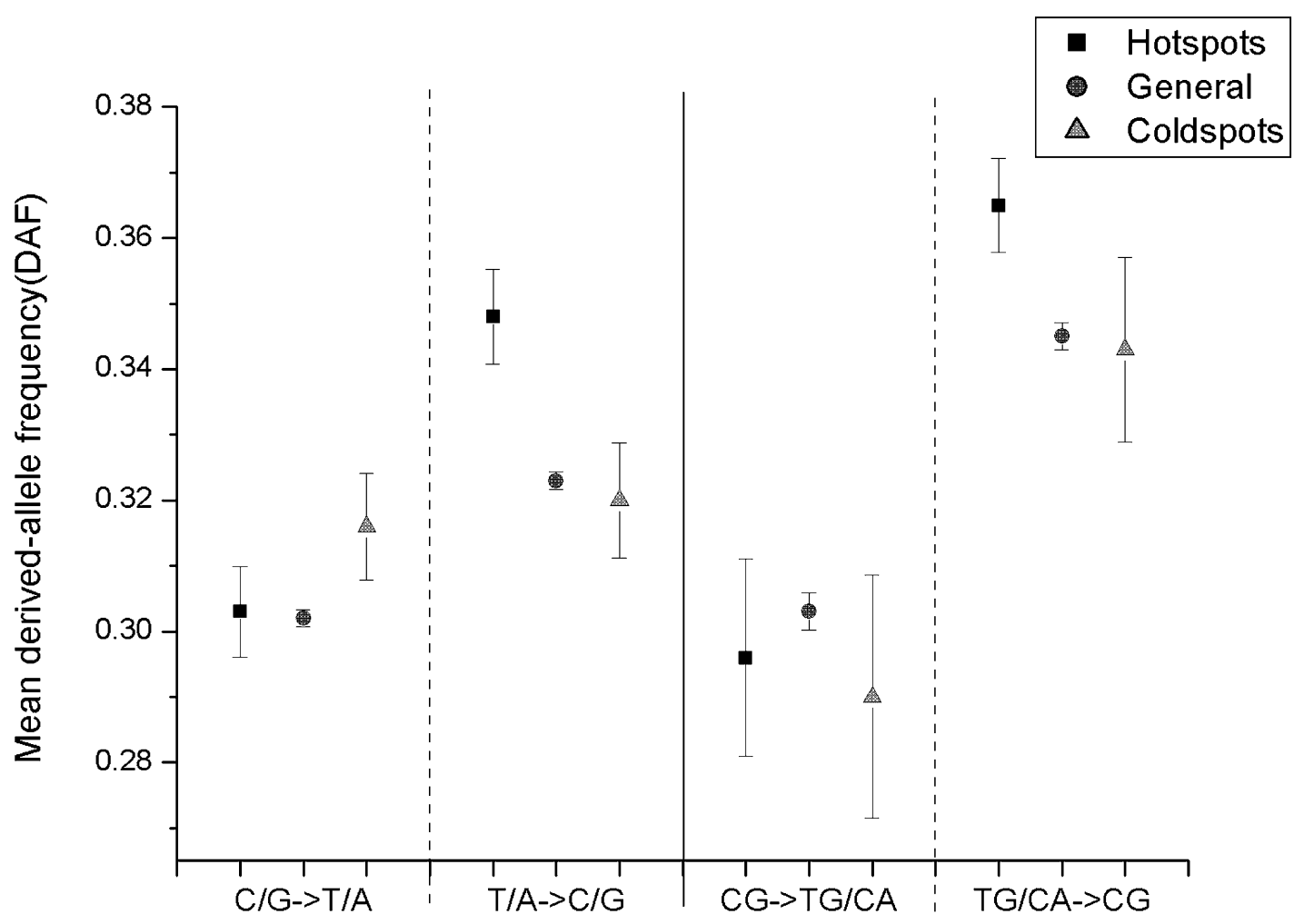

Figure 2 Derived-allele frequency for different mutation types in recombination hotspots and coldspots in YRI. Columns on the left of the solid line represent the pattern without the influence of the CG context (mutations not preceded by C or followed by G); columns on the right of the solid line represent the pattern with CG context. 
In the recombination hotspots, $\mathrm{C} / \mathrm{G}$ derived from $\mathrm{T} / \mathrm{A}$ had a much higher DAF than T/A that derived from $\mathrm{C} /$ G (0.347 vs. $0.304, \mathrm{P}<2.2 \mathrm{e}-16$, Mann-Whitney U Test), which is in accordance with BGC effect. Meanwhile, the DAF was significantly greater for CG derived from CA/ TG than for the control data $(0.365$ vs. $0.348, \mathrm{P}<2.2 \mathrm{e}-$ 16, Mann-Whitney U Test), whereas the DAF of TG/ CA derived from CG didn't differ significantly from the control data (0.296 vs. $0.304, \mathrm{P}=0.546$, Mann-Whitney $\mathrm{U}$ Test). In the recombination coldspots, T/A derived from $\mathrm{C} / \mathrm{G}$ had the same average DAF as that of $\mathrm{C} / \mathrm{G}$ derived from T/A (0.316 vs. 0.320, $\mathrm{P}=0.989$, MannWhitney U Test), indicating that the fixation bias toward $\mathrm{C} / \mathrm{G}$ disappeared in regions of low recombination, which is expected under BGC model. However, the DAF of CG derived from CA/TG is still higher than that of CA/TG derived from CG (0.343 vs. 0.290, P = 0.1717, Mann-Whitney U test), as well as that of the control data (0.343 vs. $0.320, P=0.230$, Mann-Whitney U test), but not statistically significant. Overall, these results indicate that the tendency observed in our study might be partially explained by recombination, further study is needed to address this question.

\section{Discussion}

\section{Mutation and fixation bias recreating ancestral CGs}

The observation that CG dinucleotides are under-represented in vertebrate genomes has received much attention. Much evidence suggests that the observed deficiency of CG dinucleotides is caused by hypermutable methylated cytosine, which mainly exists as the CG dinucleotide in vertebrate genomes[24]. This is further supported by the observation that $90 \%$ of the mutations from CGs are transitions to CA/TG in our study, accompanied with the overrepresentation of TG/CA in the genome reported by others [24]. More directly, when the CGs in CpG islands become methylated, CGs mutate to TG/CA at a comparatively high rate [37]. It is estimated that the advent of heavily methylated genomes dates to approximately 450 million years ago, leading to a lower CG frequency [38,39]. Meanwhile, CpG islands which were thought to lack methylation were also being lost $[15,17,18]$.

Yet the methylated CG dinucleotide acts as an essential substrate for many regulatory reactions [8]. This was believed to be a high fidelity process, until studies revealed the possibility of deleterious mutations induced by deamination of 5 -methylcytosine [12-14]. The question then arose as to whether CG dinucleotides would vanish from the genome.

In our study, by analyzing the mutations involving CG dinucleotides in non- $\mathrm{CpG}$ island regions, a strong tendency toward increasing CG dinucleotides was observed, due to biased mutation and fixation patterns.
Specifically, there are about three times as many mutations generating new CGs as mutations eliminating CGs, mainly due to the overwhelming number of mutations from CA/TG to CG. Furthermore, transition-generated CGs had a higher average DAF than transversion-generated CGs, and in comparison to control data, transition-generated CGs had a significantly higher DAF while transversion-generated CGs had a significantly lower DAF. Since most CA/TG dinucleotides are derived from deamination of 5-methylcytosine, many mutations from CA/TG to CG should be back-mutations after CG mutated to CA/TG. This scenario thereby recreates ancestral CGs in the genome while preventing novel CGs that derived from CC/CT/AG/ GG.

By contrast, in CpG island regions, which are relatively abundant in CG dinucleotides, there seems to be a tendency toward reducing the number of CG dinucleotides. This may reflect evolution from a non-equilibrium GC content in such regions, as the equilibrium $\mathrm{GC}$ content in the human genome is estimated to be $33 \%-42 \%[29]$, which is much lower than the present GC content (> 55\%).

\section{Natural selection or neutral mechanism?}

Although the preference for transition-generated CGs was observed both in intergenic and genic regions, it could be driven by natural selection as many regulatory elements are located in intergenic regions [40-43]. The assumption of the selection test we applied is that, if the higher average DAF was caused by positive selection on these transition-generated CGs, a larger proportion of the CGs should exhibit signals of positive selection, compared with the control dataset. However, no difference between the CG and control data was observed, indicating that newlygenerated CGs were not excessively subject to recent positive selection (Table 2). The analysis of inferred back-mutations similarly indicated that such backmutations also were not significantly influenced by recent positive selection.

It should be noted that the failure to detect the signal of recent positive selection could also have other explanations. The present methods for detecting the signal of recent positive selection are mainly targeted at a region rather than a specific locus $[33,44]$, and identifying causal variants is quite challenging. Since positive signals may come from hitchhiking with the selected allele, applying such methods to isolated sites rather than a genomic region may lack sufficient power. Moreover, weak selection would be difficult to detect as well. Therefore, these results do not rule out some role for selection in producing the patterns observed in this study. 


\section{Extension of BGC model?}

As an alternative to selection, the BGC model is a neutral process that increases the probability of fixation of mutations that increase GC content in the human genome $[20,45]$. It is therefore of interest to investigate if our findings can be explained via an extension of the BGC model in the context of CG dinucleotides, in particular the fixation preference for recreating ancestral CGs, or if some other mechanism must be invoked.

Recombination was reported to be the cause of BGC $[29,46]$ and this is supported in our study, as the fixation preference for $\mathrm{C} / \mathrm{G}$ rather than $\mathrm{A} / \mathrm{T}$ exists in recombination hotspots, but not in the recombination coldspots. The fixation preference for transition-generated CGs we observed in our study might be partially driven by the recombination, as there were no significant difference between recombination hotspots and coldspots. However, more evidence is needed to address this question, as we have a rather small sample size in this analysis.

\section{Conclusion}

In this study, we identified a trend that could be responsible for generating new CG dinucleotides in non-CpG islands, which are otherwise deficient in the human genome; namely, an excess number of mutations from CA/ TG to CG, which in turn recreates ancestral CG dinucleotides in the human genome that had been previously lost by mutation. It appears that neutral process, which might be partly associated with recombination, is responsible for this trend. By contrast, in CpG island regions, which are relatively abundant in CG dinucleotides, a tendency toward reducing the number of CG dinucleotides was observed. Overall, our results shed further light on the fate of CG dinucleotides in the genome.

\section{Additional material}

Additional file 1: Illustration of the reliability of the parsimony method to count the mutations at CpG related sites using three outgroups: chimpanzee/baboon/rhesus. The phylogeny of the four species used to infer mutations is shown in (a). Branch length indicates the rate of substitution per site at non-CpG positions. Substitution rates at $\mathrm{CpG}$ sites are 10 times higher than that at non-CpG sites, (b) scenario when the parsimony method is reliable, (c) scenario when the parsimony method is not reliable. Scenario (b) is 10 times more likely to be observed than scenario (c). Comparably, if chimpanzee, baboon, or rhesus is used as a single outgroup respectively, the probability ratio between (b) and (c) would be 2:1, 0.4:1, or 0.4:1.

Additional file 2: Derived-allele frequency estimation in CpG island regions.

Additional file 3: Number of SNPs and derived-allele frequency for different mutation types in different annotation categories in CEU. Additional file 4: Number of SNPs and derived-allele frequency for different mutation types in different annotation categories in ASN Additional file 5: Derived-allele frequency for different mutation types in CEU.
Additional file 6: Derived-allele frequency for different mutation types in ASN.

Additional file 7: Number of SNPs and derived-allele frequency for different mutation types in the $9.4 \mathrm{Mb}$ non-genic region.

Additional file 8: Derived-allele frequency estimated by different methods of inferring the mutation direction in the 9.4 Mb DNA alignment. Diamond symbol denotes the mean DAFs ( \pm s.e.m).

Additional file 9: Phylogenetic tree for reliable back-mutations from CA to $C G$

Additional file 10: Derived-allele frequency for different mutation types in recombination hotspots and coldspots in CEU.

Additional file 11: Derived-allele frequency for different mutation types in recombination hotspots and coldspots in ASN.

\section{Acknowledgements}

We thank Marcos Antezana for helpful discussion and the guidance for part of the study, Matthew Webster, Cizhong Jiang, Shiheng Tao for providing valuable advice, Mark Stoneking for revising the manuscript as a native English speaker and helpful suggestions, and Ryan D. Hernandez for providing extra information about the method to correct the allelefrequency spectrum.

\section{Author details}

${ }^{1}$ CAS-MPG Partner Institute of Computational Biology, Shanghai Institutes of Biological Sciences, Chinese Academy of Sciences, 200000, Shanghai, PR China. ${ }^{2}$ Department of Evolutionary Genetics, Max Planck Institute for Evolutionary Anthropology, D04103, Leipzig, Germany.

\section{Authors' contributions}

LMK designed the study. LMK collected the data. LMK analyzed data. LMK and CS wrote the manuscript. All authors read and approved the final manuscript.

Received: 1 July 2010 Accepted: 5 January 2011

Published: 5 January 2011

\section{References}

1. Yen PH, Patel P, Chinault AC, Mohandas T, Shapiro L: Differential methylation of hypoxanthine phosphoribosyltransferase genes on active and inactive human X chromosomes. Proc Natl Acad Sci USA 1984, 81(6):1759-1763.

2. Ferguson-Smith AC, Sasaki H, Cattanach BM, Surani MA: Parental-originspecific epigenetic modification of the mouse H19 gene. Nature 1993, 362(6422):751-755

3. Jones PA, Baylin SB: The fundamental role of epigenetic events in cancer. Nat Rev Genet 2002, 3(6):415-428.

4. Weber M, Hellmann I, Stadler MB, Ramos L, Paabo S, Rebhan M, Schubeler D: Distribution, silencing potential and evolutionary impact of promoter DNA methylation in the human genome. Nat Genet 2007, 39(4):457-466.

5. Suzuki MM, Kerr ARW, De Sousa D, Bird A: CpG methylation is targeted to transcription units in an invertebrate genome. Genome Research 2007, 17(5):625-631.

6. Wilson GG, Murray NE: Restriction and modification systems. Annu Rev Genet 1991, 25:585-627.

7. Barlow DP: Methylation and imprinting: from host defense to gene regulation? Science 1993, 260(5106):309-310.

8. Laird PW, Jaenisch R: The role of DNA methylation in cancer genetic and epigenetics. Annu Rev Genet 1996, 30:441-464.

9. Riggs AD: $X$ chromosome inactivation, differentiation, and DNA methylation revisited, with a tribute to Susumu Ohno. Cytogenet Genome Res 2002, 99(1-4):17-24.

10. Chen RZ, Pettersson U, Beard C, Jackson-Grusby L, Jaenisch R: DNA hypomethylation leads to elevated mutation rates. Nature 1998, 395(6697):89-93. 
11. Chen ZX, Riggs AD: Maintenance and regulation of DNA methylation patterns in mammals. Biochem Cell Biol 2005, 83(4):438-448.

12. Kangaspeska S, Stride B, Metivier R, Polycarpou-Schwarz M, Ibberson D, Carmouche RP, Benes V, Gannon F, Reid G: Transient cyclical methylation of promoter DNA. Nature 2008, 452(7183):112-115.

13. Metivier R, Gallais R, Tiffoche C, Le Peron C, Jurkowska RZ, Carmouche RP, Ibberson D, Barath P, Demay F, Reid G, et al: Cyclical DNA methylation of a transcriptionally active promoter. Nature 2008, 452(7183):45-50.

14. Brown TC, Jiricny J: Different base/base mispairs are corrected with different efficiencies and specificities in monkey kidney cells. Cell 1988, 54(5):705-711

15. Antequera F, Bird A: Number of $\mathrm{CpG}$ islands and genes in human and mouse. Proc Natl Acad Sci USA 1993, 90(24):11995-11999.

16. Larsen F, Gundersen G, Lopez R, Prydz H: CpG islands as gene markers in the human genome. Genomics 1992, 13(4):1095-1107.

17. Matsuo K, Clay O, Takahashi T, Silke J, Schaffner W: Evidence for erosion of mouse $\mathrm{CpG}$ islands during mammalian evolution. Somat Cell Mol Genet 1993, 19(6):543-555.

18. Jiang C, Han L, Su B, Li WH, Zhao Z: Features and trend of loss of promoter-associated $\mathrm{CpG}$ islands in the human and mouse genomes. Mol Biol Evol 2007, 24(9):1991-2000

19. Antequera F: Structure, function and evolution of $\mathrm{CpG}$ island promoters Cell Mol Life Sci 2003, 60(8):1647-1658.

20. Galtier N, Piganeau G, Mouchiroud D, Duret L: GC-content evolution in mammalian genomes: the biased gene conversion hypothesis. Genetics 2001, 159(2):907-911.

21. Hendrich B, Hardeland U, Ng HH, Jiricny J, Bird A: The thymine glycosylase MBD4 can bind to the product of deamination at methylated CpG sites. Nature 1999, 401(6750):301-304.

22. Neddermann P, Jiricny J: The Purification of a Mismatch-Specific Thymine-DNA Glycosylase from Hela-Cells. J Biol Chem 1993, 268(28):21218-21224

23. Li MK, Gu L, Chen SS, Dai JQ, Tao SH: Evolution of the isochore structure in the scale of chromosome: insight from the mutation bias and fixation bias. J Evol Biol 2008, 21(1):173-182.

24. Bird AP: DNA methylation and the frequency of CpG in animal DNA. Nucleic Acids Res 1980, 8(7):1499-1504.

25. Jiang C, Zhao Z: Mutational spectrum in the recent human genome inferred by single nucleotide polymorphisms. Genomics 2006, 88(5):527-534

26. Hernandez RD, Williamson SH, Bustamante CD: Context dependence, ancestral misidentification, and spurious signatures of natural selection. Mol Biol Evol 2007, 24(8):1792-1800.

27. Giannelli F, Anagnostopoulos T, Green PM: Mutation rates in humans. II. Sporadic mutation-specific rates and rate of detrimental human mutations inferred from hemophilia B. Am J Hum Genet 1999, 65(6):1580-1587

28. Zhang Z, Schwartz S, Wagner L, Miller W: A greedy algorithm for aligning DNA sequences. J Comput Biol 2000, 7(1-2):203-214.

29. Meunier J, Duret $\mathrm{L}$ : Recombination drives the evolution of GC-content in the human genome. Mol Biol Evol 2004, 21(6):984-990

30. Steiper ME, Young NM: Primate molecular divergence dates. Mol Phylogenet Evol 2006, 41(2):384-394

31. Takai $\mathrm{D}$, Jones PA: Comprehensive analysis of $\mathrm{CpG}$ islands in human chromosomes 21 and 22. Proc Natl Acad Sci USA 2002, 99(6):3740-3745

32. Takai $\mathrm{D}$, Jones PA: The $\mathrm{CpG}$ island searcher: a new WWW resource. In Silico Biol 2003, 3(3):235-240.

33. Voight BF, Kudaravalli $S$, Wen $X$, Pritchard JK: A map of recent positive selection in the human genome. PLoS Biol 2006, 4(3):e72.

34. Myers S, Bottolo L, Freeman C, McVean G, Donnelly P: A fine-scale map of recombination rates and hotspots across the human genome. Science 2005, 310(5746):321-324

35. Consortium TIH: A haplotype map of the human genome. Nature 2005, 437(7063):1299-1320.

36. Marais G: Biased gene conversion: implications for genome and sex evolution. Trends Genet 2003, 19(6):330-338.

37. Bird AP, Taggart MH, Nicholls RD, Higgs DR: Non-methylated CpG-rich islands at the human alpha-globin locus: implications for evolution of the alpha-globin pseudogene. Embo J 1987, 6(4):999-1004

38. Cooper DN, Krawczak M: Cytosine methylation and the fate of CpG dinucleotides in vertebrate genomes. Hum Genet 1989, 83(2):181-188.
39. Sved J, Bird A: The expected equilibrium of the CpG dinucleotide in vertebrate genomes under a mutation model. Proc Natl Acad Sci USA 1990, 87(12):4692-4696.

40. Strichman-Almashanu LZ, Lee RS, Onyango PO, Perlman E, Flam F, Frieman MB, Feinberg AP: A genome-wide screen for normally methylated human $\mathrm{CpG}$ islands that can identify novel imprinted genes. Genome Res 2002, 12(4):543-554.

41. Song F, Smith JF, Kimura MT, Morrow AD, Matsuyama T, Nagase $H$, Held WA: Association of tissue-specific differentially methylated regions (TDMs) with differential gene expression. Proc Natl Acad Sci USA 2005, 102(9):3336-3341.

42. He X, Chang S, Zhang J, Zhao Q, Xiang H, Kusonmano K, Yang L, Sun ZS, Yang $\mathrm{H}$, Wang J: MethyCancer: the database of human DNA methylation and cancer. Nucleic Acids Res 2008, 36 Database: D836-841.

43. Rauch TA, Zhong X, Wu X, Wang M, Kernstine KH, Wang Z, Riggs AD, Pfeifer GP: High-resolution mapping of DNA hypermethylation and hypomethylation in lung cancer. Proc Natl Acad Sci USA 2008, 105(1):252-257.

44. Sabeti PC, Varilly P, Fry B, Lohmueller J, Hostetter E, Cotsapas C, Xie X, Byrne EH, McCarroll SA, Gaudet R, et al: Genome-wide detection and characterization of positive selection in human populations. Nature 2007, 449(7164):913-918.

45. Webster MT, Smith NG, Hultin-Rosenberg L, Arndt PF, Ellegren H: Maledriven biased gene conversion governs the evolution of base composition in human alu repeats. Mol Biol Evol 2005, 22(6):1468-1474.

46. Montoya-Burgos Jl, Boursot P, Galtier N: Recombination explains isochores in mammalian genomes. Trends Genet 2003, 19(3):128-130.

doi:10.1186/1471-2148-11-3

Cite this article as: Li and Chen: The tendency to recreate ancestral CG dinucleotides in the human genome. BMC Evolutionary Biology 2011 11:3.

\section{Submit your next manuscript to BioMed Central and take full advantage of:}

- Convenient online submission

- Thorough peer review

- No space constraints or color figure charges

- Immediate publication on acceptance

- Inclusion in PubMed, CAS, Scopus and Google Scholar

- Research which is freely available for redistribution

Submit your manuscript at www.biomedcentral.com/submit
C) Biomed Central 